\title{
Application and Research on Affection Model Based on Bayesian Network
}

\author{
Lin Shi, Zhiliang Wang, and Zhigang Li \\ ${ }^{1}$ P.O. BOX 135, University of Science and Technology, Beijing 100083, China \\ ${ }^{2}$ Computer Center, Tangshan College, Tangshan, Hebei 063000, China \\ \{pattiesl, lzghello\}@126.com, \\ wzl@263.net
}

\begin{abstract}
It needs not only intelligence but also emotion for the computer to realize harmonious human computer interaction, which is one of the research focuses in the field of computer science. This paper proposes a hierarchical approach to represent personality, affection and emotion, using Bayesian Network to affection model and show emotion via virtual human's facial expression. The affection model was applied to an affective HCI system, proved to be simple, effective and stable.
\end{abstract}

\section{Hierarchical Model of the Virtual Human}

\subsection{Instruction of the Hierarchical Model}

We construct a hierarchical model: The Personality-affection-emotion model. Based on OCEAN model in psychology field, We classify human's personality into five dimensions: 1] Openness, Conscientiousness, Extraversion, Agreeableness, and Neuroticism; each factor corresponds to one dimension in the personality space 2, and each dimension is closely-related with facial expression and affection representation. We classify affection into positive and negative [3] adopting the most popular classification of basic emotions: happiness, surprise, fear, sadness, disgust, and anger, in addition, we add a neutral emotion. Corresponding to emotions, we use Ekman's theory, six basic facial expressions [4 [5] [6] and another neutral facial expression.

\subsection{Extension of the AIML Tag}

We take the chatting robot ALICE as our virtual human, which is based on AIML (Artificial Intelligence Markup Language) Technology. When inputs a question, it will produce a relative answer. There are detailed descriptions about AIML in literature [7. In order to endow ALICE with emotion, we add an emotion tag to represent her response emotion. There are seven emotion tags corresponding to seven basic emotions mentioned above. For example(5\% probability of sad, $95 \%$ probability of happy): 
$<$ category>

<pattern>How are you doing nowadays?</pattern>

$<$ template><emo name="happiness" prob="95">

<emo name="sadness" prob="5"> Everything is running smoothly.

$</$ template $>$

$</$ category $>$

\section{Construction of the Affection Model Based on Bayesian Network}

As Fig.1 shows, we construct an affection model based on Bayesian network involving two parent nodes and one child node, one corresponding model for each personality factor of the OCEAN model. Of course, user can combine any two or several factors arbitrarily .For example, user can totally constructs such a personality: $20 \%$ openness and $80 \%$ Neuroticism, the value range of "Current Affection $A_{c}$ " and "Response Affection $A_{r}$ " in Fig.1 is either positive or negative. Initial value of $A_{c}$ depends on different personality. $A_{r}$ is extracted from

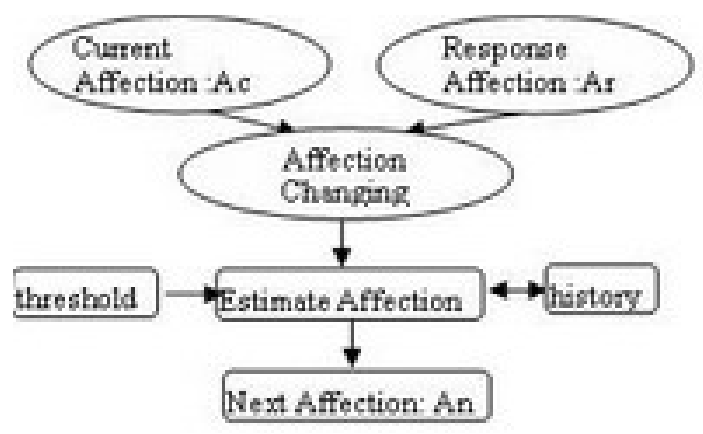

Fig. 1. Affection model for each personality

the emotion tags of ALICE's answer. There are different conditional transition probabilities for each personality $\pi$ to decide next affection. The probability represents affective process.

We get the conditional probability for the changing of affection $P\left(A_{n} \mid A_{c}, A_{r}\right)$ after training to ALICE with different personality. Once the conditional transition probability and prior probability $P\left(e_{i}\right)$ are given, a possible affection can be definite according to the following formula:

$$
P\left(A_{n}\right)=B B N\left(A_{c}, A_{r}, \pi\right)=P\left(A_{n} \mid A_{c}, A_{r}\right) P\left(e_{i}\right)
$$

$P\left(A_{n}\right)$ decides the affection change. When $P\left(A_{n}\right)>k($ a threshold, $0 \leq k \leq 1)$, we choose $A_{n}$ as the next affection state. Otherwise, hold the previous affection state. The history preserves $P\left(A_{n}\right)$ for the next computation. 


\section{Conversion from Affection to Emotion}

When the next affection state is certain, it's necessary to choose qualifying emotion response to control the virtual human's facial expression. There are three key factors deciding the emotion state: ALICE's response emotion $e_{r}$, current affection $A_{n}$ (output of the affection model) and the previous emotion state, defined as $e_{p}$.

The first key factor can be easily controlled by adding emotion tags in ALICE's answers when we establish AIML database. The second key-factor is the output of the affection model. As for positive affection and negative affection, we defined emotion state transition probability matrix respectively and experientially. More users testing the system can certainly optimize these values, getting more believable results. Formula (2) shows how to get the next emotion state $e_{n}$ :

$$
P\left(e_{n}\right)=\Gamma A_{n}\left(\operatorname{Ex}\left(e_{p}\right), \operatorname{Ex}\left(e_{r}\right)\right) P\left(e_{r}\right)
$$

$\Gamma A_{n}$ denotes the transition probability matrix of affection $A_{n}$. $E x\left(e_{r}\right)$ denotes the corresponding expression. we also set a threshold $s,(0 \leq s \leq 1)$. Only if $P\left(e_{n}\right) \geq s$, the emotion state changes to $e_{n}$, otherwise, it will remain unchanging.

\section{Results}

We hypothesize Personality:70\% Openness, 30\% Neuroticism, affection is positive. So we can get $\Gamma A_{n}$ experientially.

$$
\Gamma A_{n}=\left[\begin{array}{cccc}
0.8 & 0.2 & 0.05 & 0.05 \\
0.6 & 0.2 & 0.1 & 0.1 \\
0.7 & 0.05 & 0.05 & 0.2 \\
0.3 & 0.05 & 0.15 & 0.3
\end{array}\right]
$$

The user and virtual human play roles as student and teacher respectively; Four basic emotions , m1,m2,m3,m4 denotes happiness, angry, sadness and neutral respectively; And there's a regulation if the maximal probability is $10 \%$ bigger than the second maximal probability, then don't consider the influence of the latter.The threshold $s=0.5$, virtual teacher's emotion is $e_{r}$.The followings are results of the experiment based on the above assumption:

(1) An happy conversation. Input :"Good morning Ms Yang!", virtual teacher is influenced by positive emotion, after training and testing lots of times, we can get the corresponding probability: $P\left(e_{r}\right)=\left[\begin{array}{llll}0.8 & 0.05 & 0.1 & 0.05\end{array}\right]^{T}$, according to the proposed formula for the emotion probability, we can get the results:

$$
P\left(e_{n}\right)=\Gamma A_{n} \cdot P\left(e_{r}\right)=\left[\begin{array}{llll}
0.6525 & 0.505 & 0.5775 & 0.2775
\end{array}\right]^{T}
$$

After some judges, the next emotion is happiness. Then choose the facial expression corresponding to happiness according to $E x\left(e_{p}\right)$. The system result is as left part of Fig.2 shows.

(2)An unhappy conversation. Input : "Ms Yang, I forgot doing my homework yesterday!", virtual teacher is influenced by negative emotion, after training 
and testing lots of times, we can get the corresponding probability: $P\left(e_{r}\right)=$ $\left[\begin{array}{llll}0.05 & 0.5 & 0.2 & 0.25\end{array}\right]^{T}$, according to the proposed formula for the emotion probability, we can get the results:

$$
P\left(e_{n}\right)=\Gamma A_{n} \cdot P\left(e_{r}\right)=\left[\begin{array}{llll}
0.1125 & 0.175 & 0.12 & 0.145
\end{array}\right]^{T}
$$

After some judges, the next emotion is anger. Then choose the facial expression corresponding to anger according to $\operatorname{Ex}\left(e_{p}\right)$. The system result is as right part of Fig.2 shows.
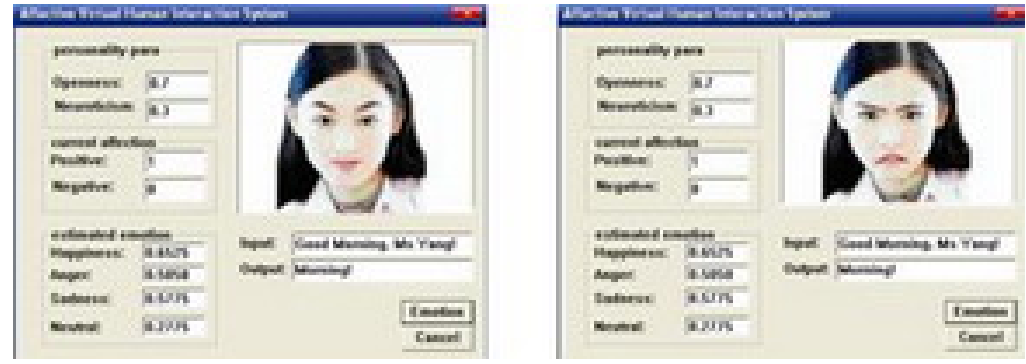

Fig. 2. Affective HCI System Interface, Emotion is happiness and anger

Acknowledgments. Work supported by Chinese National Nature Science Foundation (No.60573059) and by Foundation of University of Science and Technology Beijing.

\section{References}

1. Costa, P.T., McCrae, R.R.: Normal personality assessment in clinical practice. The NEO personality inventory, Psychological Assessment (1992)

2. Chen, M., Luo, J., Dong, S.: ATOM-A task oriented multi-channel interface structure model. Journal of Computer-Aided Design \& Computer Graphics, 61-67 (1996)

3. Deng, L., Zheng, R.: Affect Variables and Mental Health in College Students. Chinese Mental Health Journal 18, 58-60 (2004)

4. Li, D., Guo, D.: The Study of Self-Organization in Emotional System. Advances in Psychological Science 12(4), 512-518 (2004)

5. Morishima, S.: Realtime Face Analysis and Synthesis Using Neural Network. In: Neural Networks for Signal Processing X, pp. 13-22. IEEE Press, Los Alamitos (2000)

6. Suzuki, K., Yamada, H., Hartono, P., Hashimoto, S.: Modeling of Interrelationship between Physical Feature of Face and Its Impression. In: Proc. The First Int'l Symposium on Measurement, Analysis and Modeling of Human Functions, pp. 310-315 (2001)

7. Xue, W., Shi, Z., Gu, X., Wang, Z.: The Research of Emotional Interaction System Based on Agent. Computer Engineering and Applications 38(19), 6-8 (2002) 